Check for updates
OPEN ACCESS

\section{Association of soy and fermented soy product intake with total and cause specific mortality: prospective cohort study}

\author{
Ryoko Katagiri, ${ }^{1}$ Norie Sawada, ${ }^{1}$ Atsushi Goto, ${ }^{1}$ Taiki Yamaji, ${ }^{1}$ Motoki Iwasaki, ${ }^{1}$ Mitsuhiko Noda, ${ }^{2}$ \\ Hiroyasu Iso, ${ }^{3}$ Shoichiro Tsugane ${ }^{1}$, for the Japan Public Health Center-based Prospective Study \\ Group
}

${ }^{1}$ Epidemiology and Prevention Group, Center for Public Health Sciences, National Cancer Centre, 5-1-1 Tsukiji, Chuo-ku, ${ }^{2}$ Department of Diabetes, Metabolism and Endocrinology, Ichikawa Hospital, International University of Health and Welfare, Ichikawa, Chiba, Japan ${ }^{3}$ Public Health, Department of Social Medicine, Osaka University Graduate School of Medicine, Suita-shi, Osaka, Japan

Correspondence to: N Sawada nsawada@ncc.go.jp

(ORCID 0000-0002-9936-1476) Additional material is published online only. To view please visit the journal online.

Cite this as: BMJ 2020;368:m34 http://dx.doi.org/10.1136/bmj.m34

Accepted: 16 December 2019 Tokyo 104-0045, Japan

\section{ABSTRACT}

OBJECTIVE

To investigate the association between several types of soy products and all cause and cause specific mortality.

\section{DESIGN}

Population based cohort study.

SETTING

Japan Public Health Centre-based Prospective Study, which includes 11 public health centre areas in Japan. PARTICIPANTS

92915 participants (42 750 men and 50165 women) aged 45 to 74 years.

\section{EXPOSURES}

Intake of total soy products, fermented soy products (natto and miso), non-fermented soy products, and tofu from a five year survey questionnaire.

\section{MAIN OUTCOME MEASURES}

All cause and cause specific mortality (cancer, total cardiovascular disease, heart disease, cerebrovascular disease, respiratory disease, and injury) obtained from residential registries and death certificates.

\section{RESULTS}

During 14.8 years of follow-up, 13303 deaths were identified. In the multivariable adjusted models, intake of total soy products was not significantly associated with total mortality. Compared with the lowest fifth of total soy product intake, the hazard ratios in the highest fifth were 0.98 (95\% confidence interval 0.91 to $1.06, P_{\text {trend }}=0.43$ ) in men and 0.98 (0.89 to $\left.1.08, P_{\text {trend }}=0.46\right)$ in women. Intake of fermented soy products was inversely associated with all cause mortality in both sexes (highest versus lowest fifth: 0.90 (0.83 to 0.97$), P_{\text {trend }}=0.05$ in men, and 0.89 ( 0.80 to 0.98$), P_{\text {trend }}=0.01$ in women). Natto showed significant and inverse associations with total cardiovascular disease related mortality in both sexes.

\section{WHAT IS ALREADY KNOWN ON THIS TOPIC}

Findings on the association between intake of total soy products and all cause mortality are inconsistent

\section{WHAT THIS STUDY ADDS}

In this large prospective study conducted in Japan with a high rate of soy consumption, no significant association was found between intake of total soy products and all cause mortality

In contrast, a higher intake of fermented soy products (natto and miso) was associated with a lower risk of mortality

\section{CONCLUSIONS}

In this study a higher intake of fermented soy was associated with a lower risk of mortality. A significant association between intake of total soy products and all cause mortality was not, however, observed. The findings should be interpreted with caution because the significant association of fermented soy products might be attenuated by unadjusted residual confounding.

\section{Introduction}

Diet is a determinant of non-communicable diseases, which are responsible for more than $70 \%$ of deaths globally. ${ }^{1}$ One risk factor for such diseases is the shift from intake of traditional, plant based food to energy dense, high fat diets. ${ }^{2}$ The intake of soy products, which are still consumed in large amounts in Asian countries, ${ }^{3}$ might improve the nutrient intake levels in people with a low consumption of plant based food. ${ }^{45}$

Traditional Asian diets contain several types of processed soy products. Natto (soybeans fermented with Bacillus subtilis), miso (soybeans fermented with Aspergillus oryzae), tofu (soybean curd), and abura-age (fried tofu) are widely consumed in Japan (see supplementary table 1). Recent prospective studies have shown that intake of fermented soy products, not total soy intake, is associated with a lower risk of high blood pressure ${ }^{6}$ and that intake of natto (a fermented soy product) is associated with a reduction in cardiovascular disease related mortality. ${ }^{7}$ However, few epidemiological studies have focused on the effect of fermented soy product intake on mortality from causes other than cardiovascular disease. Moreover, two prospective studies that investigated the association between intake of total soy products and all cause mortality presented inconsistent data. ${ }^{89}$ Components of soy, such as isoflavone and fibre, were shown to have anticancer, anticardiovascular disease, cholesterol lowering, and weight loss effects. ${ }^{10-15}$ Because fermented soy products are made from whole soy beans, the characteristics of fermented soy products are a small loss of these nutrients and an abundance of bioactive components such as polyamine, ${ }^{16}$ or nattokinase in natto. ${ }^{17}$

We conducted a large scale prospective study on intake of total and fermented soy products in Japan with follow-up duration of approximately 15 years. Our aim was to investigate the association between intake of several types of soy products and all cause and cause specific mortality. 


\section{Methods}

Study design

The Japan Public Health Centre-based Prospective Study comprises two cohorts: in the first cohort the baseline survey was initiated in 1990 and in the second cohort it was initiated in $1993 .^{18}$ We conducted follow-up surveys at five and 10 years. Since the five year follow-up food frequency questionnaire includes more detailed information on diet than our baseline questionnaire, ${ }^{19}$ we used data from the five year survey as the baseline variables of this study.

\section{Settings}

We conducted the Japan Public Health Centrebased Prospective Study in 11 public health centres nationwide. The first cohort concerned five public health centre areas and the second concerned six. The five year survey, which was set as baseline in the present analyses, commenced in 1995 in the first cohort and in 1998 in the second cohort. At this point we collected questionnaires to obtain updated information on dietary habits, lifestyle, and health status. We followed eligible participants from the submission date of the five year survey to 31 December 2012, except for one Tokyo area in which participants were followed until 31 December 2009. Participants were followed until date of death, emigration overseas, or the last day of the follow-up period, whichever came first.

\section{Participants}

The first cohort comprised participants aged 45 to 64 years residing in five public health centre areas, whereas the second cohort comprised participants aged 45 to 74 years living in six public health centre areas at the time of the five year survey. Participants who responded to the five year questionnaires were eligible for participation in this study (response rate $76.2 \%)$.

We followed death or migration of participants using the residential registry of the public health centre areas. Information on cause of death was obtained from death certificates with permission of the Japanese Ministry of Health, Labour, and Welfare.

\section{Variables}

Exposures were intake of total soy products, fermented soy products (including natto and miso), and nonfermented soy products (including tofu). The main outcomes were all cause and cause specific mortality from five main causes of death in Japan: cancer, total cardiovascular diseas (including heart disease and cerebrovascular disease), respiratory disease, and injury. Other variables included body mass index (BMI), smoking status, alcohol intake, leisure time physical exercise or sport, self reported history of diabetes or use of drugs for diabetes, use of antihypertensives, consumption of coffee and green tea, health checkups, menopause status in women, exogenous female hormone use in women, and dietary intakes such as total energy intake and intake of vegetables, fruit, fish, and meat.

\section{Data sources and measurements}

The five year food frequency questionnaire included 138 foods and beverages. Participants were asked about the frequency of intake and portion size for each item consumed over the previous year (see supplementary table 1$).{ }^{19}$ To calculate miso intake (g/ day) we multiplied the amount of miso soup consumed by 0.08 because this soup comprises about $92 \%$ water and $8 \%$ miso based on its standard recipe. The validity of the questionnaire was assessed using a dietary record kept by a subsample of the cohort for 14 or 28 days. Assessments of the validity and reproducibility for nutrient data acquired using the food frequency questionnaire have been reported previously. ${ }^{20} 21$ Spearman's correlation coefficients between the energy adjusted intake of soy products from the food frequency questionnaire and those from dietary records were 0.53 in men and 0.49 in women from the first cohort and 0.52 and 0.54 , respectively, from the second cohort. ${ }^{20}$ ${ }^{21}$ Reproducibility between the estimates for soy foods that were one year apart were 0.64 in the first cohort and 0.57 in the second cohort for men and 0.67 and 0.44 , respectively, for women. ${ }^{2122}$

We traced death or migration of participants using the residential registry of the public health centre areas. Information on cause of death was obtained from death certificates with permission of the Japanese Ministry of Health, Labour, and Welfare. ICD-10 codes (international classification of diseases and related health problems, 10th revision) were assigned to causes of death. All cause mortality and five main causes of death were assessed: cancer (codes C00-C97), total cardiovascular disease mortality (I00-I99), heart disease (I20-I52), cerebrovascular disease (I60-I69), respiratory disease (J10-J18 and J40-J47), and injury (V01-Y09 and Y85-Y86).

Self reported anthropometric data, information on lifestyle, and medical and smoking histories were assessed with a self administered questionnaire as part of the five year survey.

\section{Quantitative variables}

We used the residual method to adjust the amount of soy products for total energy intake. The amount of total soy products was the sum of consumed tofu, yushidofu, koyadofu, abura-age, natto, soy milk, and miso (calculated from miso soup). Intakes of fermented soy products were calculated as the sum of natto and miso, whereas non-fermented soy products included tofu, yushidofu, koyadofu, abura-age, and soy . The intake of total soy products, fermented soy products, non-fermented soy products, natto, miso, and tofu (that is, the sum of tofu, yushidofu, and koyadofu) were incorporated into the individual statistical model. We divided participants into fifths of intake for each of these products, except for natto, by sex, and the lowest category was used as the reference. As more than 12000 participants answered that they did 
not eat natto, the first fifth of natto intake comprised those who did not eat natto ( $\mathrm{n}=12450$ men and 12227 women); the remaining participants were divided into quarters.

\section{Statistical analysis}

Hazard ratios and corresponding 95\% confidence intervals were calculated for the association between soy product intake and all cause and cause specific mortality using Cox proportional hazards regression models. We estimated $\mathrm{P}$ for trend values with regression models in which ordinal values for each category of soy products were used as continuous variables. Models were adjusted for potential confounding factors. Model 1 included age at the five year survey (continuous) and public health centre area. Model 2 was additionally adjusted for BMI at the five year survey $(<21,21-\leq 23$, $23-25$, and $>25 \mathrm{~kg} / \mathrm{m}^{2}$ ), smoking status (never, former, $<20$ cigarettes/day, and $\geq 20$ cigarettes/day), alcohol intake (never, occasional, <150 g/week, and $\geq 150 \mathrm{~g} /$ week), leisure time physical exercise or sports (almost never, $\geq$ once/month, and $\geq 3$ times/week), self reported history of diabetes or taking drugs for diabetes (yes or no), taking antihypertensives (yes or no), consumption of coffee and green tea (almost never, $>1$ cup/week, $>1$ cup/day, $>2$ cups/day, and $>4$ cups/day), undergoing health check-ups (yes or no), menopause status in women (premenopausal or postmenopausal), exogenous female hormone use in women (yes or no), total energy intake (fifths), and energy adjusted amount of vegetables, fruit, fish, and meat (fifths). We further adjusted for area deprivation index, developed previously-this indicator reflects neighbourhood, rather than individual, deprivation level. ${ }^{23} 24$ We conducted the same analyses after the exclusion of participants who died during the first three years. Overall, $278(0.3 \%)$ study participants were lost to follow-up during the period. For them, we treated the last confirmed date as the censoring date. We calculated age adjusted and area adjusted cumulative mortality rate at 10 years in each fifth of soy product intake using the PROC PHREG BASELINE statement (SAS Institute, Cary, NC). Multiple imputations of missing values were performed for covariates (BMI, smoking, alcohol intake, physical exercise, coffee intake, green tea intake, use of exogenous female hormones (women only), and menopausal status (women only)) using multivariate normal imputation. All covariates, followup durations, and mortality statuses were included in the model for imputation using the SAS PROC MI procedure. ${ }^{25}$ We conducted 10 rounds of multiple imputations, then combined them into final estimates according to Rubin's rule (SAS PROC MIANALYZE procedure). ${ }^{25} \mathrm{P}$ values were two sided, with $\mathrm{P}<0.05$ considered statistically significant. Statistical analyses were conducted using the SAS software, version 9.4. (SAS Institute, Cary, NC).

\section{Patient and public involvement}

Patients were not involved in the study design, implementation, or setting the research questions or the outcome measures directly. Participants in the Japan Public Health Centre-based Prospective Study are community residents and we have held regular meetings with health practitioners in study areas to obtain opinions on health practice since the beginning of this study.

\section{Results}

Figure 1 shows the number of eligible participants and participants analysed in this study. Participants with a self reported history of cancer, stroke, or myocardial infarction during the five year period or with a history of these diseases were excluded ( $n=4634)$ as were 5923 participants who reported extreme energy intakes (ie, $<2.5$ and $>97.5$ centiles). In total, 92915 participants (42750 men and 50165 women) were included in the analysis. Table 1 presents the baseline characteristics of the participants, divided into fifths of total soy product consumption, and the number of those with missing values. Among both sexes, those with a higher consumption of soy products tended to be older, have a higher BMI, and be less likely to be current smokers. Supplementary tables 2 to 4 show the characteristics of the participants divided into fifths of intake of fermented soy product, natto, and miso, respectively. Participants with a higher intake of fermented soy products or miso were older, whereas the trend of age according to the categories of natto consumption was U-shaped. The percentage of current smokers was lower in the highest category of fermented soy or natto intake than that in the lowest category, whereas

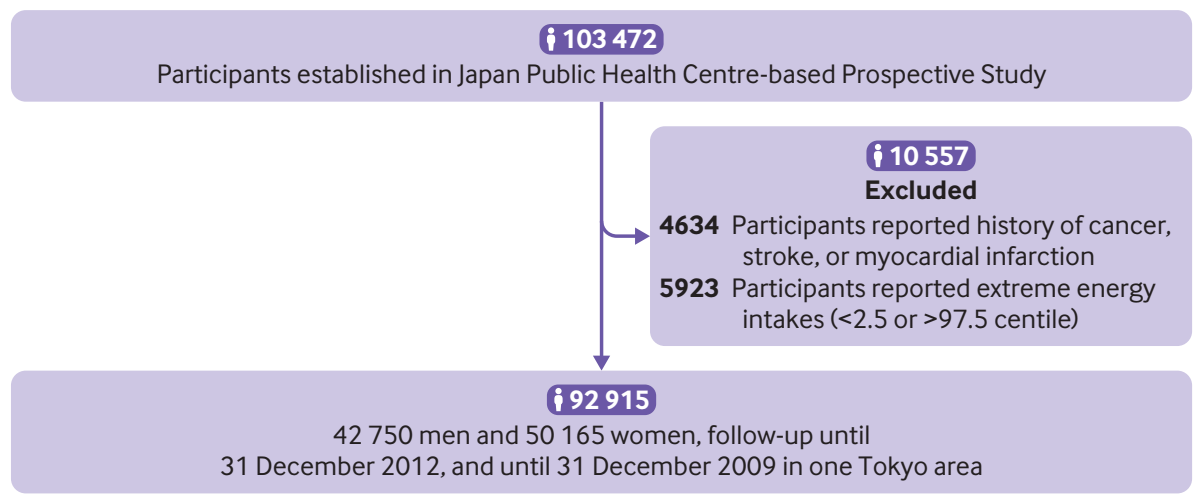

Fig 1 | Flow of participants through study 


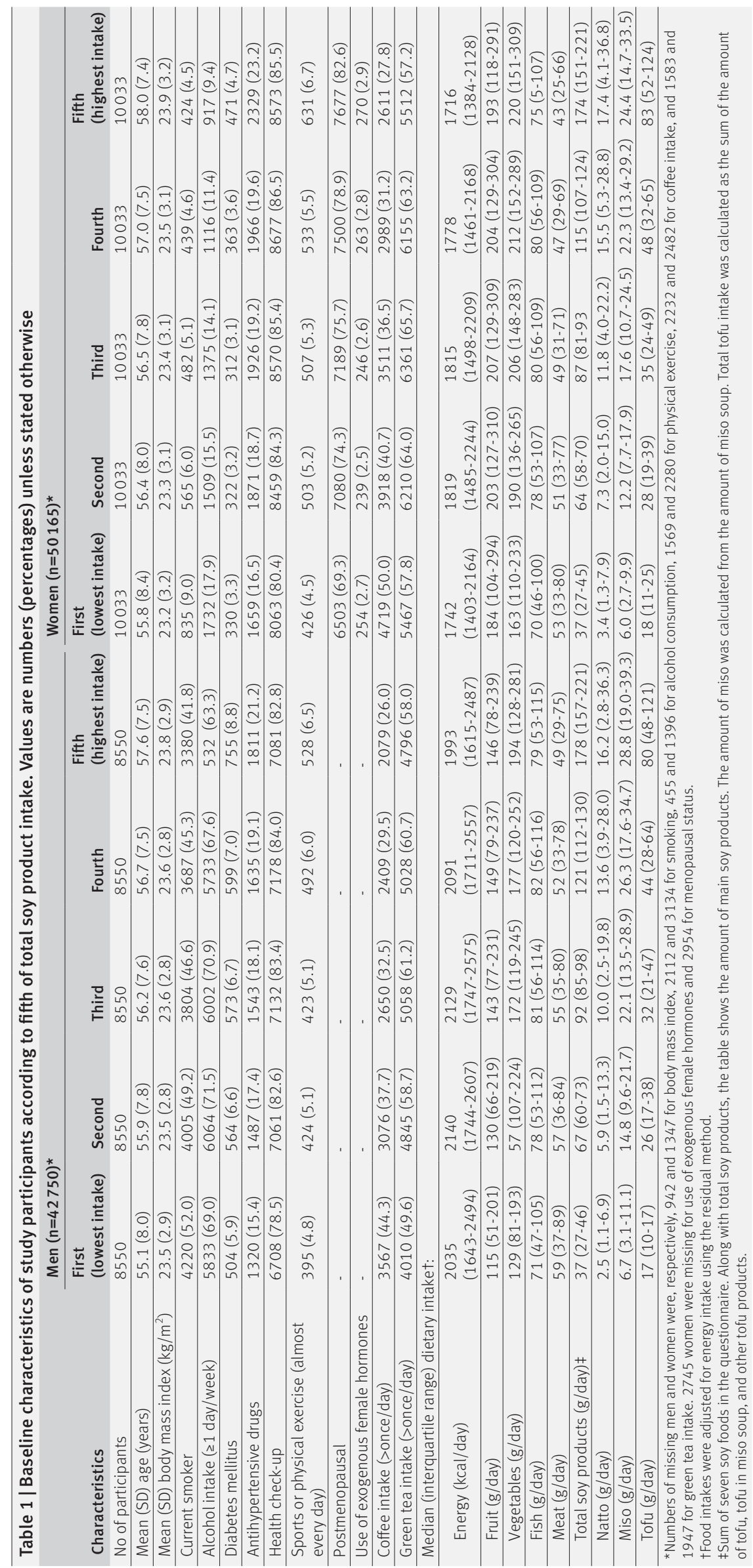




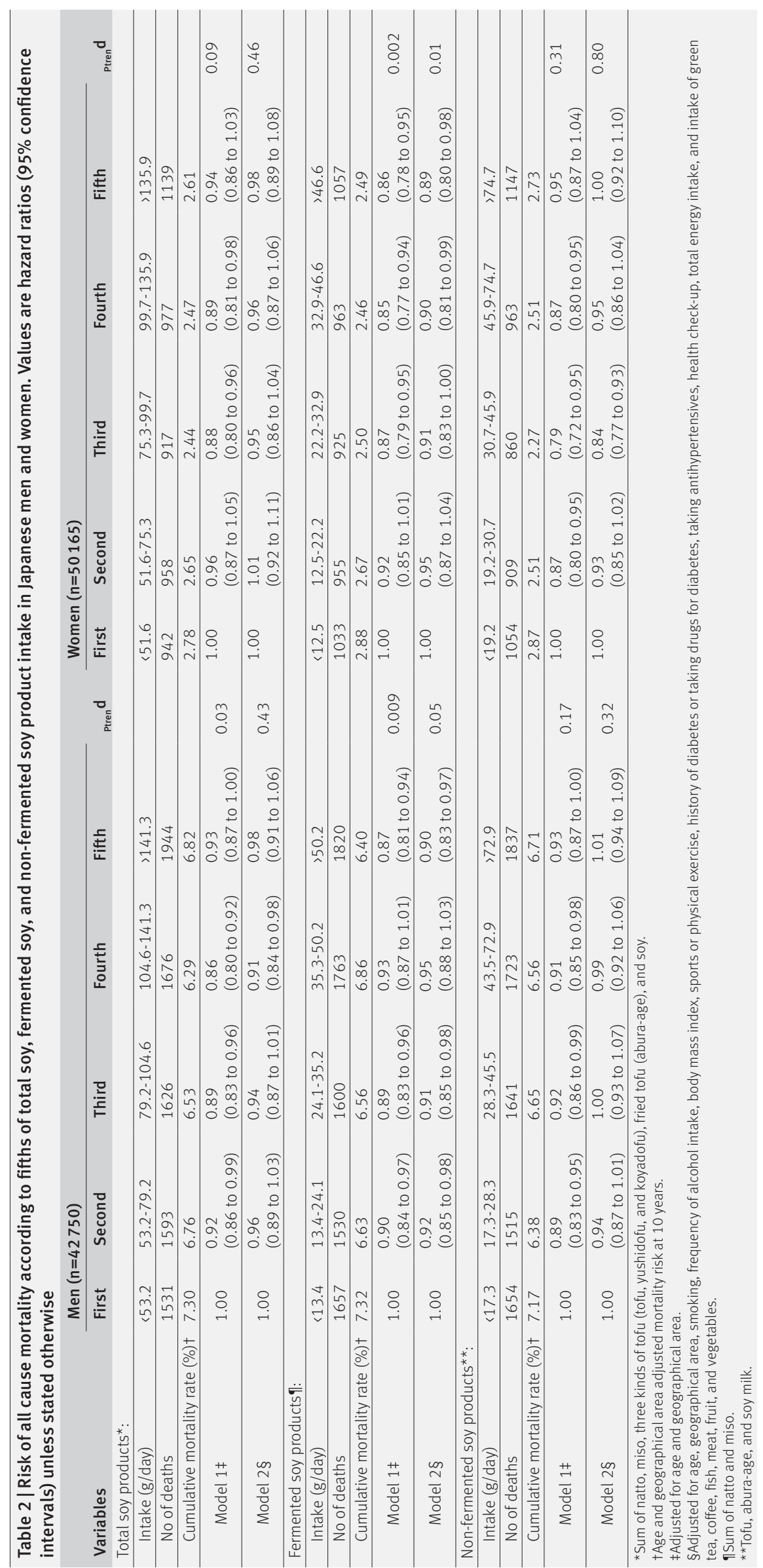

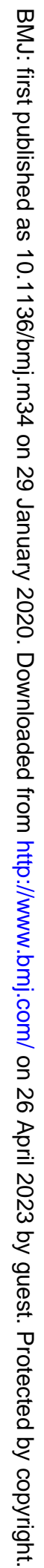


a higher percentage of current smokers was observed in the highest category of miso intake compared with the lowest category in men. The mean follow-up period was 14.8 years, and the total person years were 1374643 , with 13303 reported deaths (8370 men and 4933 women).

\section{All cause mortality}

Table 2 shows the hazard ratios and corresponding 95\% confidence intervals for all cause mortality according to the amount of consumed total, fermented, and non-fermented soy products. In both sexes, total soy product intake was marginally inversely associated with all cause mortality after adjustment for age and area. After further adjustment for potential confounding factors, the associations were attenuated. Compared with the lowest fifth of intake, the hazard ratios in the highest fifth were 0.98 (95\% confidence interval 0.91 to $1.06, \mathrm{P}_{\text {trend }}=0.43$ ) in men and 0.98 (0.89 to $\left.1.08, \mathrm{P}_{\text {trend }}=0.46\right)$ in women. Fermented soy intake was inversely associated with all cause death after adjusting for potential confounding factors. Compared with the lowest fifth of intake, the hazard ratios in the highest fifth were $0.90(0.83$ to $\left.0.97, \mathrm{P}_{\text {trend }}=0.05\right)$ in men and 0.89 (0.80 to 0.98, $\left.P_{\text {trend }}=0.01\right)$ in women. Intake of non-fermented soy products was not significantly associated with all cause mortality.

Table 3 shows the associations between all cause mortality and consumption of soy products (natto, miso, and tofu). The intake of soy products was not significantly associated with total mortality in men, whereas in women intakes of natto and miso were inversely associated with all cause mortality (hazard ratios in the highest fifth of intake were 0.84 $\left(\mathrm{P}_{\text {trend }}=0.001\right)$ for natto and $0.89\left(\mathrm{P}_{\text {trend }}=0.03\right)$ for miso $)$.

\section{Cause specific mortality}

Tables 4 and 5 show the hazard ratios for cause specific mortality according to fifth of soy product intake for men and women, respectively. The intakes of all kinds of soy products were not significantly associated with cancer related mortality. A significant association commonly observed was the inverse association between natto intake and total cardiovascular disease related mortality (ICD-10: I00-I99) in both men and women (hazard ratios for the highest fifths of intake were 0.76 ( 0.65 to $\left.0.90, \mathrm{P}_{\text {trend }}=0.002\right)$ in men and 0.79 ( 0.65 to $\left.0.95, \mathrm{P}_{\text {trend }}=0.01\right)$ in women). Increased intake of fermented soy products was significantly associated with decreased total cardiovascular disease related mortality in men (hazard ratio for the highest fifth of intake was $0.82\left(0.70\right.$ to $\left.\left.0.97, \mathrm{P}_{\text {trend }}=0.04\right)\right)$; this association was not significant in women $(0.89$ ( 0.73 to 1.07, $\left.\mathrm{P}_{\text {trend }}=0.25\right)$ ). Supplemental tables 5 and 6 show the results of the sensitivity analyses. The associations between intake of fermented soy products and all cause mortality were marginal after the exclusion of patients who died within the first three years (hazard ratios for the highest fifth of intake were 0.90 (0.83 to 0.98, $\left.\mathrm{P}_{\text {trend }}=0.13\right)$ in men and 0.91 ( 0.82 to $1.01, \mathrm{P}_{\text {trend }}=0.05$ in women)). The results were similar when the model was further adjusted for area deprivation index.

\section{Discussion}

This large prospective study investigated the association between intake of several kinds of soy products and mortality. The findings showed that the consumption of total soy products was not significantly associated with a decrease in all cause mortality, whereas intake of fermented soy products was significantly inversely associated with all cause mortality in both sexes. The risk of mortality in the highest fifth of fermented soy intake, including natto and miso, was 10\% lower than that in the lowest fifth. Intake of non-fermented soy products was not significantly associated with all cause mortality. Although a significant reduction in mortality was observed, our findings should be interpreted with caution because unadjusted residual confounding might remain and attenuate the association of fermented soy products. Moreover, other caution is needed because these associations were shown epidemiologically and do not translate directly to clinical benefit.

\section{Strengths and limitations of this study}

The main strengths of this study are its prospective design, relatively large sample size, high response rate, low rate of loss to follow-up, and long duration of follow-up; these attributes allowed for the recording of the main causes of death. Information on deaths was obtained from the registries of each participating public health centre, thereby limiting potential bias. The Japan Public Health Centre-based Prospective Study includes populations from various areas in Japan, encompassing individuals with a wide range of soy product intake, and the obtained data allowed for the investigation of the association between the intake of a variety of soy products and mortality.

Our study does have several limitations. The most important one was that we could not eliminate residual unmeasured confounding completely. Although we adjusted for known and measured factors and excluded known diseases diagnosed before baseline, such as cancer, stroke, or myocardial infarction, we could not adjust for socioeconomic status variables other than the area deprivation index or diseases other than diabetes and hypertension. It could be speculated that the higher prevalence of diabetes or hypertension in the high consumption group might reflect an accurate diagnosis of individuals in these groups. According to our previous study, ${ }^{26}$ however, although the positive predictive value of self reported diabetes was $94 \%$ and specificity was $99 \%$ in our study, around $4 \%$ of participants were unaware of their diabetes status and did not answer that they had diabetes in the survey questionnaire but were identified by laboratory data. $^{27}$ Thus, although people without self reported diabetes might be a cause of residual confounding, it is implausible that the prevalence of diabetes in each fifth of soy product intake influenced the mortality in our study. In addition, soy based foods could have 


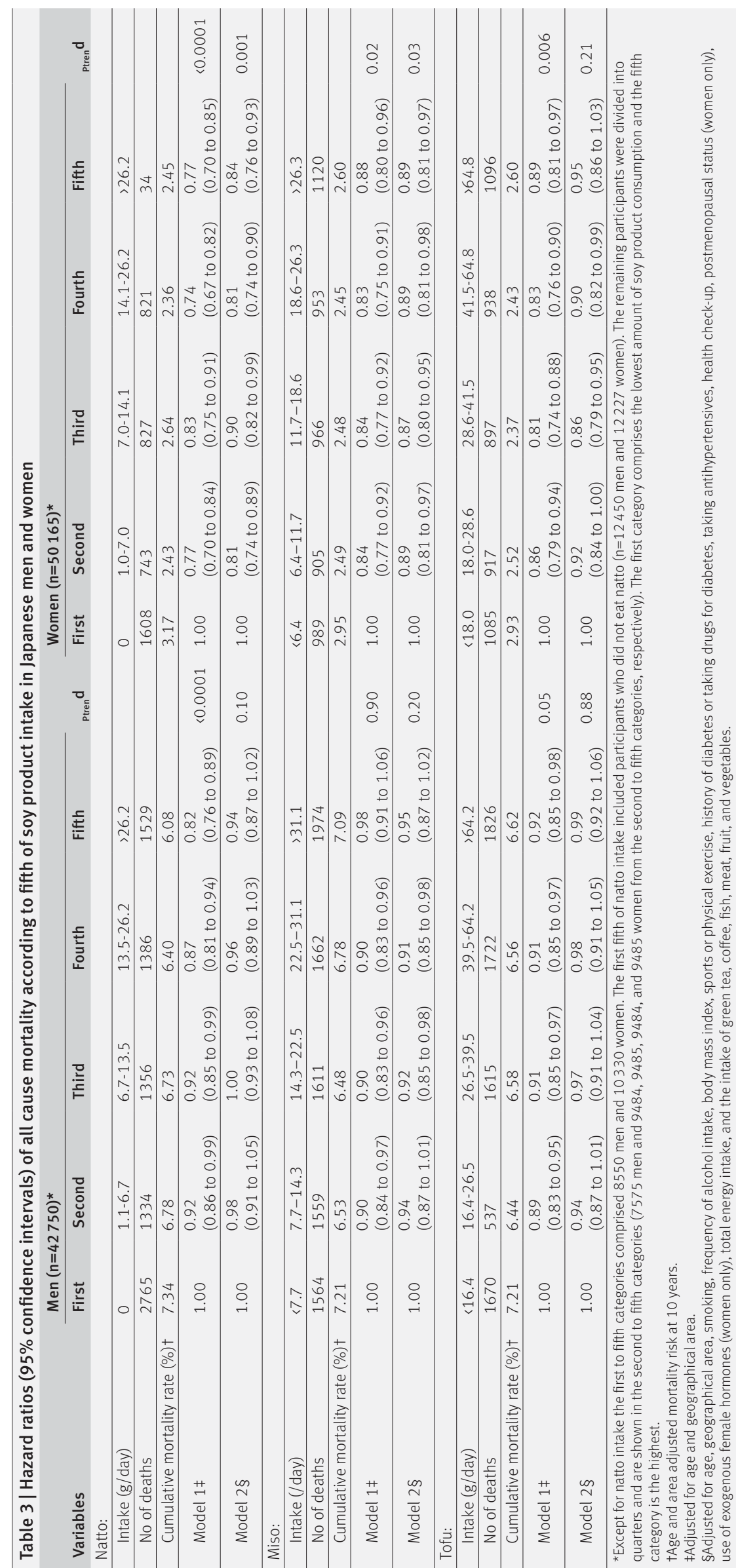




\begin{tabular}{|c|c|c|c|c|c|c|}
\hline \multirow[b]{2}{*}{ Causes of death, by soy product } & \multicolumn{5}{|l|}{$\operatorname{Men}(n=42750)^{*}$} & \multirow[b]{2}{*}{ Ptren $d$} \\
\hline & First (lowest intake) & Second & Third & Fourth & Fifth (highest intake) & \\
\hline \multicolumn{7}{|l|}{ Cancer } \\
\hline Total soy products & 582 & 616 & 652 & 693 & 777 & \\
\hline Model 2 & 1 & 0.97 (0.86 to 1.08$)$ & 1.00 (0.89 to 1.12$)$ & $1.01(0.90$ to 1.13$)$ & 1.09 (0.96 to 1.22$)$ & 0.10 \\
\hline Fermented soy products & 647 & 593 & 624 & 731 & 725 & \\
\hline Model 2 & 1 & 0.91 (0.81 to 1.02$)$ & $0.92(0.82$ to 1.03$)$ & $1.04(0.93$ to 1.17$)$ & 0.97 (0.85 to 1.09$)$ & 0.66 \\
\hline Non-fermented soy & 616 & 622 & 652 & 721 & 709 & \\
\hline Model 2 & 1 & $1.00(0.90$ to 1.13$)$ & 1.04 (0.93 to 1.17$)$ & $1.10(0.98$ to 1.23$)$ & 1.06 (0.94 to 1.19$)$ & 0.12 \\
\hline Natto & 601 & 619 & 656 & 667 & 777 & \\
\hline Model 2 & 1 & 1.01 (0.90 to 1.13$)$ & 0.97 (0.86 to 1.10$)$ & $1.00(0.89$ to 1.13$)$ & 0.98 (0.87 to 1.11$)$ & 0.74 \\
\hline Miso & 601 & 619 & 656 & 667 & 777 & \\
\hline Model 2 & 1 & 0.95 (0.85 to 1.07$)$ & 0.97 (0.86 to 1.08$)$ & 0.95 (0.84 to 1.06$)$ & $1.02(0.91$ to 1.16$)$ & 0.74 \\
\hline Tofu & 618 & 629 & 655 & 680 & 738 & \\
\hline Model 2 & 1 & 1.01 (0.90 to 1.13$)$ & 1.04 (0.93 to 1.17$)$ & $1.03(0.92$ to 1.16$)$ & 1.09 (0.97 to 1.22$)$ & 0.13 \\
\hline \multicolumn{7}{|l|}{ Total cardiovascular disease } \\
\hline Total soy products & 345 & 397 & 398 & 397 & 463 & \\
\hline Model 2 & 1 & 1.03 (0.89 to 1.20$)$ & 0.97 (0.84 to 1.13$)$ & $0.89(0.77$ to 1.04$)$ & $0.95(0.82$ to 1.11$)$ & 0.18 \\
\hline Fermented soy products & 363 & 369 & 424 & 436 & 408 & \\
\hline Model 2 & 1 & 0.98 (0.84 to 1.14$)$ & $1.04(0.90$ to 1.21$)$ & $1.00(0.85$ to 1.17$)$ & $0.82(0.70$ to 0.97$)$ & 0.04 \\
\hline Non-fermented soy & 419 & 348 & 386 & 396 & 451 & \\
\hline Model 2 & 1 & 0.85 (0.74 to 0.98$)$ & $0.91(0.79$ to 1.04$)$ & $0.87(0.75$ to 1.00$)$ & 0.95 (0.82 to 1.09$)$ & 0.63 \\
\hline Natto & 691 & 306 & 317 & 342 & 344 & \\
\hline Model 2 & 1 & 0.86 (0.74 to 1.00$)$ & $0.86(0.73$ to 1.00$)$ & 0.85 (0.73 to 0.99$)$ & $0.76(0.65$ to 0.90$)$ & 0.002 \\
\hline Miso & 345 & 365 & 380 & 437 & 473 & \\
\hline Model 2 & 1 & 0.96 (0.84 to 1.13$)$ & 0.95 (0.81 to 1.10$)$ & $1.04(0.90$ to 1.21$)$ & 0.95 (0.82 to 1.10$)$ & 0.84 \\
\hline Tofu & 425 & 348 & 381 & 406 & 440 & \\
\hline Model 2 & 1 & $0.83(0.72$ to 0.96$)$ & 0.89 (0.77 to 1.02$)$ & $0.87(0.75$ to 1.00$)$ & $0.90(0.78$ to 1.04$)$ & 0.30 \\
\hline \multicolumn{7}{|l|}{ Heart disease } \\
\hline Total soy products & 189 & 229 & 209 & 202 & 239 & \\
\hline Model 2 & 1 & $1.08(0.88$ to 1.31$)$ & $0.90(0.74$ to 1.11$)$ & 0.79 (0.64 to 0.98$)$ & $0.82(0.67$ to 1.02$)$ & 0.004 \\
\hline Fermented soy products & 201 & 203 & 221 & 233 & 210 & \\
\hline Model 2 & 1 & $0.98(0.81$ to 1.20$)$ & 0.99 (0.81 to 1.22$)$ & 0.97 (0.78 to 1.19$)$ & 0.77 (0.62 to 0.97$)$ & 0.03 \\
\hline Non-fermented soy & 228 & 189 & 214 & 202 & 235 & \\
\hline Model 2 & 1 & 0.83 (0.68 to 1.00$)$ & 0.88 (0.72 to 1.07$)$ & $0.75(0.62$ to 0.92$)$ & 0.41 (0.67 to 0.99) & 0.03 \\
\hline Natto & 376 & 170 & 160 & 194 & 168 & \\
\hline Model 2 & 1 & $0.90(0.74$ to 1.10$)$ & $0.82(0.66$ to 1.02$)$ & $0.92(0.75$ to 1.14$)$ & 0.71 (0.57 to 0.88) & 0.01 \\
\hline Miso & 194 & 174 & 212 & 247 & 241 & \\
\hline Model 2 & 1 & 0.84 (0.68 to 1.03$)$ & 0.97 (0.79 to 1.18$)$ & $1.06(0.87$ to 1.30$)$ & $0.86(0.70$ to 1.06$)$ & 0.74 \\
\hline Tofu & 234 & 191 & 204 & 214 & 225 & \\
\hline Model 2 & 1 & 0.81 (0.66 to 0.98$)$ & 0.82 (0.67 to 0.99$)$ & 0.77 (0.63 to 0.94$)$ & $0.74(0.61$ to 0.90$)$ & 0.005 \\
\hline \multicolumn{7}{|l|}{ Cerebrovascular disease } \\
\hline Total soy products & 128 & 144 & 155 & 170 & 183 & \\
\hline Model 2 & 1 & $1.02(0.80$ to 1.30$)$ & $1.04(0.82$ to 1.33$)$ & $1.06(0.83$ to 1.36$)$ & $1.08(0.85$ to 1.39$)$ & 0.46 \\
\hline Fermented soy & 126 & 147 & 169 & 175 & 163 & \\
\hline Model 2 & 1 & $1.10(0.86$ to 1.40$)$ & $1.16(0.91$ to 1.49$)$ & $1.12(0.87$ to 1.44$)$ & $0.91(0.70$ to 1.20$)$ & 0.46 \\
\hline Non-fermented soy & 167 & 131 & 144 & 157 & 181 & \\
\hline Model 2 & 1 & 0.83 (0.66 to 1.05$)$ & 0.89 (0.71 to 1.12$)$ & $0.94(0.74$ to 1.18$)$ & $1.07(0.86$ to 1.34$)$ & 0.31 \\
\hline Natto & 259 & 117 & 130 & 126 & 148 & \\
\hline Model 2 & 1 & $0.84(0.66$ to 1.07$)$ & 0.89 (0.69 to 1.13$)$ & $0.78(0.61$ to 1.01$)$ & 0.83 (0.65 to 1.07$)$ & 0.14 \\
\hline Miso & 125 & 160 & 143 & 157 & 195 & \\
\hline Model 2 & 1 & $1.15(0.90$ to 1.45$)$ & $0.94(0.73$ to 1.20$)$ & 0.99 (0.78 to 1.27$)$ & $1.04(0.82$ to 1.33$)$ & 0.83 \\
\hline Tofu & 165 & 127 & 148 & 158 & 182 & \\
\hline Model 2 & 1 & $0.81(0.64$ to 1.03$)$ & 0.94 (0.74 to 1.18$)$ & $0.95(0.75$ to 1.19$)$ & $1.08(0.86$ to 1.36$)$ & 0.24 \\
\hline \multicolumn{7}{|l|}{ Respiratory disease } \\
\hline Total soy products & 99 & 102 & 110 & 123 & 184 & \\
\hline Model 2 & 1 & $0.97(0.73$ to 1.28$)$ & $1.01(0.76$ to 1.35$)$ & $1.03(0.78$ to 1.37$)$ & $1.36(1.03$ to 1.78$)$ & 0.01 \\
\hline Fermented soy products & 141 & 94 & 102 & 125 & 156 & \\
\hline Model 2 & 1 & $0.72(0.55$ to 0.95$)$ & $0.79(0.60$ to 1.03$)$ & $0.88(0.67$ to 1.15$)$ & $0.97(0.73$ to 1.28$)$ & 0.74 \\
\hline Non-fermented soy & 106 & 96 & 115 & 134 & 167 & \\
\hline Model 2 & 1 & $0.96(0.73$ to 1.27$)$ & $1.10(0.84$ to 1.44$)$ & $1.17(0.89$ to 1.53$)$ & $1.31(1.00$ to 1.70$)$ & 0.02 \\
\hline Natto & 247 & 79 & 75 & 91 & 126 & \\
\hline Model 2 & 1 & $0.82(0.62$ to 1.07$)$ & $0.82(0.61$ to 1.10$)$ & $0.88(0.66$ to 1.18$)$ & $0.96(0.73$ to 1.27$)$ & 0.90 \\
\hline Miso & 114 & 113 & 109 & 114 & 168 & \\
\hline Model 2 & 1 & $0.92(0.70$ to 1.19$)$ & $0.88(0.67$ to 1.15$)$ & $0.90(0.68$ to 1.19$)$ & $1.07(0.82$ to 1.40$)$ & 0.55 \\
\hline Tofu & 110 & 105 & 109 & 138 & 156 & \\
\hline Model 2 & 1 & $1.00(0.76$ to 1.31$)$ & $0.99(0.75$ to 1.30$)$ & 1.13 (0.87 to 1.47$)$ & $1.13(0.87$ to 1.47$)$ & 0.23 \\
\hline
\end{tabular}




\begin{tabular}{|c|c|c|c|c|c|c|}
\hline \multirow[b]{2}{*}{ Causes of death, by soy product } & \multicolumn{5}{|l|}{ Men $(n=42750)^{*}$} & \multirow[b]{2}{*}{ Ptren $d$} \\
\hline & First (lowest intake) & Second & Third & Fourth & Fifth (highest intake) & \\
\hline \multicolumn{7}{|l|}{ Injury } \\
\hline Total soy products & 137 & 123 & 144 & 131 & 149 & \\
\hline Model 2 & 1 & $0.82(0.64$ to 1.05$)$ & $0.91(0.71$ to 1.16$)$ & $0.77(0.59$ to 0.99$)$ & $0.85(0.66$ to 1.10$)$ & 0.22 \\
\hline Fermented soy products & 124 & 125 & 138 & 149 & 148 & \\
\hline Model 2 & 1 & $0.91(0.71$ to 1.18$)$ & $0.91(0.70$ to 1.18$)$ & $0.91(0.70$ to 1.19$)$ & $0.86(0.65$ to 1.13$)$ & 0.36 \\
\hline Non-fermented soy & 149 & 141 & 134 & 115 & 145 & \\
\hline Model 2 & 1 & 1.01 (0.80 to 1.28$)$ & 0.97 (0.76 to 1.23$)$ & $0.81(0.63$ to 1.04$)$ & 1.01 (0.79 to 1.29$)$ & 0.50 \\
\hline Natto & 186 & 124 & 136 & 121 & 117 & \\
\hline Model 2 & 1 & $1.10(0.86$ to 1.41$)$ & $1.17(0.90$ to 1.52$)$ & $0.99(0.75$ to 1.30$)$ & $0.96(0.72$ to 1.27$)$ & 0.53 \\
\hline Miso & 129 & 111 & 128 & 136 & 180 & \\
\hline Model 2 & 1 & $0.81(0.62$ to 1.04$)$ & $0.82(0.63$ to 1.05$)$ & $0.84(0.65$ to 1.08$)$ & $1.00(0.78$ to 1.28$)$ & 0.76 \\
\hline Tofu & 147 & 145 & 127 & 124 & 141 & \\
\hline Model 2 & 1 & 1.05 (0.83 to 1.33) & $0.93(0.73$ to 1.19$)$ & $0.89(0.69$ to 1.14$)$ & $1.00(0.78$ to 1.28$)$ & 0.57 \\
\hline
\end{tabular}

been consumed with vegetables or fruit. ${ }^{28}$ This might have led to the lower mortality in the high consumption group. Although we adjusted for the intake of food groups and measured variables, other lifestyle variables, including unmeasured factors, might be associated with mortality. Secondly, measurement errors are inherent because of the self reported nature of the food frequency questionnaire. However, these misclassifications were likely to attenuate the associations described. Thirdly, the intake of soy products was assessed at a single time point, although participants' dietary habits could have changed during the follow-up period. The Spearman's rank correlation coefficients for intake of total soy products between the five year survey and 10 year survey were 0.42 in both sexes. This dietary change might attenuate the association shown in this study. Finally, although our prospective study in a country with a relatively high consumption of soy products allowed for the investigation of the association between soy product intake and mortality, the generalisability of our findings to other populations is limited and further studies are required for replication.

Comparison with other studies, interpretation, and implication for all cause mortality

Although soy products are regularly consumed in Asia, even Asian studies focusing on the effects of soy consumption on health have been inconsistent. ${ }^{89}$ A prospective study of residents in a single city that investigated the intake of total soy products and isoflavone revealed a marginal inverse association between intake of total soy products and all cause mortality in both men and women. ${ }^{8}$ Another study showed a U-shaped association between the frequency of soy intake and death only in men, whereas intake of soy products was not significantly associated with death when both men and women were analysed together. ${ }^{9}$ Our large prospective study with a long follow-up presented a novel result, in that the intake of fermented soy products was associated with a reduced risk of overall death in men and women. Moreover, in terms of dose-response, a reduction in the mortality risk from fermented soy intake was noted between the first and second fifths on intake; this trend did not significantly change after the exclusion of participants who died within the first three years. Therefore, we suggest as a public health implication that the avoidance of a low intake of fermented soy products might lower the risk of mortality, although further studies are required to examine the clinical meanings for soy intake.

\section{Interpretation for cause specific mortality}

In our study increased consumption of fermented soy products was associated with decreased cardiovascular disease associated mortality, particularly in men. Natto intake was significantly associated with lower cardiovascular diseaseassociated mortality in both men and women. A recent Japanese study focusing on the association between natto intake and cardiovascular disease related mortality ${ }^{7}$ showed results that were consistent with ours. Although both miso and natto are fermented soy products, only natto intake showed a significant reduction in cardiovascular disease related mortality in our study, possibly because its sodium content (a risk factor for cardiovascular disease ${ }^{29}$ ) is lower than that of miso. ${ }^{30}$ Other studies that examined the association between cardiovascular disease related mortality and total soy intake or isoflavone intake showed inconsistent results. ${ }^{29} 3132$ In prospective studies including Spanish and Singaporean-Chinese participants, no associations between isoflavone or dietary soy intake and cardiovascular disease related mortality were found, ${ }^{33}$ which was attributed to lower intake of soy foods (especially those of fermented soy products) in those countries than in Japan. ${ }^{34}$

Furthermore, we found no association between soy product intake and all cancer related mortality. The association between soy intake and the incidence of several types of cancers has been investigated previously, ${ }^{10}$ and studies in both Asian and Western countries showed an association between higher soy consumption and a reduced risk of mortality in women 


\begin{tabular}{|c|c|c|c|c|c|c|}
\hline \multirow[b]{2}{*}{ Causes of death, by soy product } & \multicolumn{5}{|l|}{ Women $(n=50165)^{*}$} & \multirow[b]{2}{*}{ Ptren $d$} \\
\hline & First (lowest intake) & Second & Third & Fourth & Fifth (highest intake) & \\
\hline \multicolumn{7}{|l|}{ Cancer } \\
\hline Total soy products & 326 & 364 & 366 & 359 & 402 & \\
\hline Model 2 & 1 & $1.08(0.92$ to 1.25$)$ & 1.05 (0.90 to 1.22$)$ & $0.99(0.85$ to 1.16$)$ & 1.03 (0.88 to 1.21$)$ & 0.88 \\
\hline Fermented soy products & 356 & 367 & 343 & 365 & 386 & \\
\hline Model 2 & 1 & 1.02 (0.88 to 1.18$)$ & 0.93 (0.79 to 1.09$)$ & $0.95(0.80$ to 1.11$)$ & 0.94 (0.79 to 1.11$)$ & 0.30 \\
\hline Non-fermented soy & 352 & 357 & 347 & 355 & 406 & \\
\hline Model 2 & 1 & $1.03(0.89$ to 1.20$)$ & 0.98 (0.84 to 1.13) & $1.00(0.85$ to 1.15$)$ & $1.07(0.91$ to 1.24$)$ & 0.61 \\
\hline Natto & 527 & 290 & 311 & 336 & 353 & \\
\hline Model 2 & 1 & $0.84(0.72$ to 0.98$)$ & 0.89 (0.76 to 1.05$)$ & $0.90(0.76$ to 1.05$)$ & $0.88(0.75$ to 1.04$)$ & 0.28 \\
\hline Miso & 346 & 336 & 389 & 372 & 374 & \\
\hline Model 2 & 1 & $0.92(0.79$ to 1.07$)$ & 1.01 (0.87 to 1.17$)$ & 0.95 (0.81 to 1.11$)$ & $0.88(0.75$ to 1.03$)$ & 0.23 \\
\hline Tofu & 357 & 358 & 366 & 340 & 396 & \\
\hline Model 2 & 1 & 1.03 (0.88 to 1.19) & 1.02 (0.88 to 1.18$)$ & 0.94 (0.81 to 1.10$)$ & $1.03(0.88$ to 1.20$)$ & 0.87 \\
\hline \multicolumn{7}{|l|}{ Total cardiovascular disease } \\
\hline Total soy products & 241 & 253 & 220 & 288 & 324 & \\
\hline Model 2 & 1 & $1.04(0.87$ to 1.24$)$ & $0.86(0.71$ to 1.04$)$ & $1.06(0.89$ to 1.27$)$ & $1.03(0.86$ to 1.23$)$ & 0.64 \\
\hline Fermented soy products & 268 & 251 & 232 & 273 & 302 & \\
\hline Model 2 & 1 & 0.95 (0.80 to 1.13$)$ & $0.84(0.70$ to 1.01$)$ & $0.92(0.76$ to 1.11$)$ & 0.89 (0.73 to 1.07$)$ & 0.25 \\
\hline Non-fermented soy & 280 & 251 & 204 & 265 & 326 & \\
\hline Model 2 & 1 & 1.00 (0.84 to 1.19) & 0.77 (0.64 to 0.93) & 1.01 (0.85 to 1.21$)$ & $1.09(0.92$ to 1.29$)$ & 0.34 \\
\hline Natto & 446 & 189 & 224 & 209 & 258 & \\
\hline Model 2 & 1 & 0.76 (0.64 to 0.92$)$ & $0.87(0.72$ to 1.04$)$ & 0.72 (0.60 to 0.88$)$ & $0.79(0.65$ to 0.95$)$ & 0.01 \\
\hline Miso & 258 & 228 & 242 & 261 & 337 & \\
\hline Model 2 & 1 & 0.84 (0.70 to 1.01$)$ & 0.80 (0.67 to 0.96$)$ & 0.89 (0.74 to 1.07) & $0.94(0.79$ to 1.13$)$ & 0.86 \\
\hline Tofu & & & 49 & 70 & 58 & \\
\hline Model 2 & 1 & $0.94(0.80$ to 1.12$)$ & 0.80 (0.67 to 0.95$)$ & 0.89 (0.75 to 1.06$)$ & $0.98(0.82$ to 1.16$)$ & 0.64 \\
\hline \multicolumn{7}{|l|}{ Heart disease } \\
\hline Total soy products & 124 & 126 & 99 & 142 & 172 & \\
\hline Model 2 & 1 & 1.03 (0.80 to 1.33$)$ & 0.81 (0.62 to 1.06$)$ & 1.11 (0.86 to 1.43$)$ & 1.13 (0.88 to 1.46$)$ & 0.24 \\
\hline Fermented soy products & 146 & 121 & 115 & 136 & 145 & \\
\hline Model 2 & 1 & 0.88 (0.69 to 1.13$)$ & 0.86 (0.66 to 1.11$)$ & 0.98 (0.76 to 1.27$)$ & 0.94 (0.72 to 1.22$)$ & 0.93 \\
\hline Non-fermented soy & 141 & 120 & 90 & 143 & 169 & \\
\hline Model 2 & 1 & $0.97(0.76$ to 1.24$)$ & 0.69 (0.53 to 0.90$)$ & 1.10 (0.86 to 1.40$)$ & 1.09 (0.86 to 1.39$)$ & 0.27 \\
\hline Natto & 246 & 99 & 107 & 91 & 120 & \\
\hline Model 2 & 1 & 0.84 (0.66 to 1.08$)$ & $0.93(0.72$ to 1.20$)$ & 0.71 (0.54 to 0.94$)$ & $0.81(0.62$ to 1.06$)$ & 0.07 \\
\hline Miso & 125 & 116 & 121 & 137 & 164 & \\
\hline Model 2 & 1 & 0.89 (0.69 to 1.15$)$ & 0.85 (0.66 to 1.10$)$ & $1.05(0.82$ to 1.36$)$ & $1.03(0.80$ to 1.32$)$ & 0.46 \\
\hline Tofu & 146 & 120 & 104 & 129 & 164 & \\
\hline Model 2 & 1 & 0.94 (0.74 to 1.20$)$ & 0.77 (0.60 to 1.00) & 0.97 (0.75 to 1.24) & $1.05(0.83$ to 1.34$)$ & 0.62 \\
\hline \multicolumn{7}{|l|}{ Cerebrovascular disease } \\
\hline Total soy products & 96 & 108 & 102 & 116 & 124 & \\
\hline Model 2 & 1 & $1.08(0.81$ to 1.42$)$ & $0.93(0.70$ to 1.24$)$ & 0.98 (0.73 to 1.30$)$ & 0.91 (0.68 to 1.21$)$ & 0.37 \\
\hline Fermented soy & 97 & 113 & 100 & 112 & 124 & \\
\hline Model 2 & 1 & $1.10(0.84$ to 1.46$)$ & 0.86 (0.64 to 1.15$)$ & 0.84 (0.62 to 1.13$)$ & $0.78(0.57$ to 1.05$)$ & 0.03 \\
\hline Non-fermented soy & 115 & 107 & 93 & 104 & 127 & \\
\hline Model 2 & 1 & $1.04(0.80$ to 1.36$)$ & 0.87 (0.66 to 1.15) & $0.99(0.75$ to 1.30$)$ & 1.09 (0.83 to 1.43$)$ & 0.67 \\
\hline Natto & 164 & 75 & 103 & 98 & 106 & \\
\hline Model 2 & 1 & 0.68 (0.50 to 0.91) & 0.83 (0.63 to 1.11) & $0.69(0.52$ to 0.93$)$ & 0.67 (0.50 to 0.89$)$ & 0.02 \\
\hline Miso & 107 & 88 & 104 & 103 & 144 & \\
\hline Model 2 & 1 & 0.78 (0.59 to 1.04$)$ & $0.80(0.61$ to 1.05$)$ & 0.77 (0.58 to 1.02$)$ & 0.88 (0.67 to 1.15$)$ & 0.46 \\
\hline Tofu & 125 & 110 & 93 & 100 & 118 & \\
\hline Model 2 & 1 & 0.98 (0.76 to 1.27$)$ & $0.80(0.61$ to 1.06$)$ & 0.88 (0.67 to 1.15$)$ & $0.93(0.71$ to 1.22$)$ & 0.43 \\
\hline \multicolumn{7}{|l|}{ Respiratory disease } \\
\hline Total soy products & 59 & 65 & 55 & 44 & 74 & \\
\hline Model 2 & 1 & 1.11 (0.77 to 1.58$)$ & 0.91 (0.62 to 1.33$)$ & 0.67 (0.45 to 1.01$)$ & 0.89 (0.61 to 1.29$)$ & 0.13 \\
\hline Fermented soy products & 67 & 60 & 57 & 53 & 60 & \\
\hline Model 2 & 1 & 0.88 (0.62 to 1.25$)$ & $0.88(0.61$ to 1.26$)$ & $0.70(0.47$ to 1.04$)$ & 0.77 (0.52 to 1.15$)$ & 0.06 \\
\hline Non-fermented soy & 70 & 53 & 46 & 63 & 65 & \\
\hline Model 2 & 1 & 0.89 (0.62 to 1.27$)$ & $0.72(0.49$ to 1.05$)$ & $0.96(0.67$ to 1.37$)$ & $0.78(0.54$ to 1.13$)$ & 0.32 \\
\hline Natto & 129 & 38 & 49 & 34 & 47 & \\
\hline Model 2 & 1 & $0.64(0.43$ to 0.95$)$ & $0.83(0.57$ to 1.22$)$ & $0.50(0.32$ to 0.77$)$ & $0.62(0.41$ to 0.92$)$ & 0.009 \\
\hline Miso & 54 & 60 & 55 & 52 & 76 & \\
\hline Model 2 & 1 & $1.12(0.77$ to 1.63$)$ & 0.89 (0.61 to 1.31$)$ & 0.91 (0.61 to 1.36) & 0.96 (0.66 to 1.40$)$ & 0.55 \\
\hline Tofu & 69 & 51 & 49 & 70 & 58 & \\
\hline Model 2 & 1 & $0.86(0.60$ to 1.25$)$ & 0.79 (0.54 to 1.15$)$ & $1.10(0.78$ to 1.56$)$ & $0.74(0.50$ to 1.08$)$ & 0.40 \\
\hline
\end{tabular}




\begin{tabular}{|c|c|c|c|c|c|c|}
\hline \multirow[b]{2}{*}{ Causes of death, by soy product } & \multicolumn{5}{|l|}{ Women $(n=50165)^{*}$} & \multirow[b]{2}{*}{ Ptren $d$} \\
\hline & First (lowest intake) & Second & Third & Fourth & Fifth (highest intake) & \\
\hline \multicolumn{7}{|l|}{ Injury } \\
\hline Total soy products & 50 & 53 & 66 & 69 & 83 & \\
\hline Model 2 & 1 & $1.07(0.72$ to 1.59$)$ & $1.30(0.89$ to 1.90$)$ & $1.30(0.88$ to 1.92$)$ & 1.49 (1.01 to 2.19) & 0.03 \\
\hline Fermented soy products & 54 & 46 & 64 & 77 & 80 & \\
\hline Model 2 & 1 & $0.89(0.60$ to 1.33$)$ & $1.16(0.78$ to 1.71$)$ & $1.29(0.87$ to 1.90$)$ & $1.23(0.82$ to 1.85$)$ & 0.11 \\
\hline Non-fermented soy & 69 & 51 & 63 & 70 & 68 & \\
\hline Model 2 & 1 & $0.81(0.56$ to 1.18$)$ & 0.99 (0.69 to 1.40$)$ & $1.10(0.79$ to 1.56$)$ & $1.06(0.74$ to 1.52$)$ & 0.34 \\
\hline Natto & 88 & 46 & 47 & 62 & 78 & \\
\hline Model 2 & 1 & 0.67 (0.46 to 0.99) & 0.64 (0.43 to 0.95) & $0.77(0.53$ to 1.13$)$ & $0.97(0.67$ to 1.41$)$ & 0.68 \\
\hline Miso & 59 & 53 & 54 & 75 & 80 & \\
\hline Model 2 & 1 & $0.88(0.61$ to 1.28$)$ & $0.82(0.56$ to 1.20$)$ & $1.10(0.76$ to 1.58$)$ & $1.02(0.71$ to 1.48$)$ & 0.54 \\
\hline Tofu & 68 & 50 & 67 & 69 & 67 & \\
\hline Model 2 & 1 & 0.81 (0.56 to 1.17) & $1.09(0.77$ to 1.54$)$ & $1.11(0.78$ to 1.58$)$ & $1.06(0.74$ to 1.52$)$ & 0.33 \\
\hline
\end{tabular}

with breast cancer. ${ }^{35}{ }^{36}$ Evidence in relation to other cancers is not well established. Isoflavone is a risk factor for some cancers but not for others, so this could explain our null findings. ${ }^{3738}$

\section{Possible mechanisms}

The bioactive components of soy products might modulate their effects on mortality. Studies on the association between soy consumption and cardiovascular disease ${ }^{7} 293132$ implied important roles for nutrients such as isoflavone, fibre, and potassium. Isoflavone was found to have blood pressure reducing and lipid profile improving properties in meta-analyses, ${ }^{39} 40$ whereas soy fibre was shown to lower cholesterol levels and induce weight loss in humans. ${ }^{14} 15$ Moreover, a prospective study in the United States showed that an increase in dietary fibre from beans was associated with reduced cardiovascular disease related death among women. ${ }^{41}$ Fermented soy products are richer in fibre and potassium as well as bioactive components than their non-fermented counterparts, including tofu. The bioactive components in fermented soy products include the fibrinolytic enzyme, nattokinase (in natto), ${ }^{17}$ and polyamine. ${ }^{16}$ The polyamine spermidine was previously found to have cardioprotective effects, with intake associated with reduced morality due to heart failure. ${ }^{42}$ Therefore, bioactive components and nutrients contained in fermented soy products might have multifaceted benefits for survival.

\section{Conclusions}

In this large prospective study conducted in a country with a high level of soy consumption, we did not observe a significant association between intake of total soy products and all cause mortality. In contrast, intake of fermented soy products, including natto and miso, were significantly associated with reduced all cause mortality. Our findings should be interpreted with caution because the significant association of fermented soy products could be attenuated by unadjusted residual confounding.
We thank staff members for their contributions and efforts in conducting the survey. Members of the Japan Public Health Centrebased Prospective Study Group are listed in https://epi.ncc.go.jp/en/ jphc/781/index.html.

Contributors: ST designed the study as the principal investigator. ST, $\mathrm{NS}, \mathrm{YT}, \mathrm{MI}, \mathrm{MN}$, and $\mathrm{HI}$ conducted the survey. RK, NS, and AG drafted the plans for the data analyses. RK conducted data analysis and drafted the manuscript. All authors were involved in interpretation of the results and revision of the manuscript, and all approved the final version of the manuscript. RK and NS are guarantors. The corresponding author attests that all the listed authors meet the authorship criteria and that no others meeting the criteria have been omitted.

Funding: This study was supported by National Cancer Center research and development fund (since 2011) and a grant-in-aid for cancer research from the Japanese Ministry of Health, Labour, and Welfare (1989 to 2010). The funders had no role in the study design, data collection and analysis, decision to publish, or preparation of the manuscript.

Competing interests: All authors have completed the ICMJE uniform disclosure form at www.icmje.org/coi disclosure.pdf and declare: no support from any organisation for the submitted work; no financial relationships with any organisations that might have an interest in the submitted work in the previous three years; and no other relationships or activities that could appear to have influenced the submitted work. Ethical approval: This study was approved by the institutional review board of the National Cancer Centre, Japan (No 2001-021, 2015 085).

Data sharing: For information on how to submit an application for gaining access to Japan Public Health Centre-based Prospective Study data, follow the instructions at https://epi.ncc.go.jp/en/ jphc/805/8155.html.

The study guarantors (RK and NS) affirm that the manuscript is an honest, accurate, and transparent account of the study being reported; that no important aspects of the study have been omitted; and that any discrepancies from the study as planned have been explained.

Dissemination to participants and related patient and public communities: The results of this research were reported in newsletters for study participants and on the study website (https://epi.ncc.go.jp/ index.html) and public lectures about disease prevention have been provided based on the results.

This is an Open Access article distributed in accordance with the Creative Commons Attribution Non Commercial (CC BY-NC 4.0) license, which permits others to distribute, remix, adapt, build upon this work non-commercially, and license their derivative works on different terms, provided the original work is properly cited and the use is non-commercial. See: http://creativecommons.org/licenses/ by-nc/4.0/

1 GBD 2016 Causes of Death Collaborators. Global, regional, and national age-sex specific mortality for 264 causes of death, 1980 2016: a systematic analysis for the Global Burden of Disease 
Study 2016. Lancet 2017;390:1151-210. doi:10.1016/S01406736(17)32152-9

2 World Health Organization, Food and Agriculture Organization. Diet, nutrition and the prevention of chronic diseases. Report No. WHO technical report series 916. 2003. https://apps.who.int/iris/ bitstream/10665/42665/1/WHO TRS 916.pdf.

3 Lukito W. Candidate foods in the asia-pacific region for cardiovascular protection: nuts, soy, lentils and tempe. Asia Pac J Clin Nutr 2001;10:128-33. doi:10.1046/j.1440-6047.2001.00240.x

4 Tucker KL, Qiao N, Maras JE. Simulation with soy replacement showed that increased soy intake could contribute to improved nutrient intake profiles in the U.S. population. J Nutr 2010;140:2296S-301S. doi:10.3945/jn.110.123901

5 Reinwald S, Akabas SR, Weaver CM. Whole versus the piecemeal approach to evaluating soy. J Nutr 2010;140:2335S-43S. doi:10.3945/in.110.124925

6 Nozue M, Shimazu T, Sasazuki S, et al. Fermented soy product intake is inversely associated with the development of high blood pressure: The Japan Public Health Center-Based Prospective Study. J Nutr 2017;147:1749-56. doi:10.3945/jn.117.250282

7 Nagata C, Wada K, Tamura T, et al. Dietary soy and natto intake and cardiovascular disease mortality in Japanese adults: the Takayama study. Am / Clin Nutr 2017:105:426-31. doi:10.3945/ajcn.116.137281

8 Nagata C, Takatsuka N, Shimizu H. Soy and fish oil intake and mortality in a Japanese community. Am J Epidemiol 2002;156:82431. doi:10.1093/aje/kwf118

9 Yamasaki K, Kayaba K, Ishikawa S. Soy and soy products intake, all-cause mortality, and cause-specific mortality in Japan: The Jichi Medical School Cohort Study. Asia Pac J Public Health 2015;27:53141. doi:10.1177/1010539514539545

10 Messina MJ, Persky V, Setchell KD, Barnes S. Soy intake and cancer risk: a review of the in vitro and in vivo data. Nutr Cancer 1994:21:113-31. doi:10.1080/01635589409514310

11 Sacks FM, Lichtenstein A, Van Horn L, Harris W, Kris-Etherton P, Winston M, American Heart Association Nutrition Committee. Soy protein, isoflavones, and cardiovascular health: an American Heart Association Science Advisory for professionals from the Nutrition Committee. Circulation 2006;113:1034-44. doi:10.1161/ CIRCULATIONAHA.106.171052

12 Kokubo Y, Iso H, Ishihara J, Okada K, Inoue M, Tsugane S, JPHC Study Group. Association of dietary intake of soy, beans, and isoflavones with risk of cerebral and myocardial infarctions in Japanese populations: the Japan Public Health Center-based (JPHC) study cohort I. Circulation 2007;116:2553-62. doi:10.1161/ CIRCULATIONAHA.106.683755

13 Zamora-Ros R, Jiménez C, Cleries R, et al. Dietary flavonoid and lignan intake and mortality in a Spanish cohort. Epidemiology 2013;24:726-33. doi:10.1097/EDE.0b013e31829d5902

$14 \mathrm{Hu} \mathrm{X,} \mathrm{Gao} \mathrm{J,} \mathrm{Zhang} \mathrm{Q} \mathrm{et} \mathrm{al.} \mathrm{Soy} \mathrm{fiber} \mathrm{improves} \mathrm{weight} \mathrm{loss} \mathrm{and} \mathrm{lipid}$ profile in overweight and obese adults: a randomized controlled trial. Mol Nutr Food Res 2013;57:2147-54. doi:10.1002/ mnfr.201300159

15 Slavin J. Nutritional benefits of soy protein and soy fiber. J Am Diet Assoc 1991;91:816-9

16 Okamoto A, Sugi E, Koizumi Y, Yanagida F, Udaka S. Polyamine content of ordinary foodstuffs and various fermented foods. BiosCi Biotechnol Biochem 1997;61:1582-4. doi:10.1271/bbb.61.1582

17 Sumi H, Hamada H, Tsushima H, Mihara H, Muraki H. A novel fibrinolytic enzyme (nattokinase) in the vegetable cheese Natto; a typical and popular soybean food in the Japanese diet. Experientia 1987;43:1110-1. doi:10.1007/BF01956052

18 Tsugane S, Sawada N. The JPHC study: design and some findings on the typical Japanese diet. Jpn / Clin Oncol 2014:44:777-82. doi:10.1093/jico/hyu096

19 Sasaki S, Kobayashi M, Ishihara J, Tsugane S, JPHC. Self-administered food frequency questionnaire used in the 5 -year follow-up survey of the JPHC Study: questionnaire structure, computation algorithms, and area-based mean intake. J Epidemiol 2003;13(Suppl):S13-22. doi:10.2188/jea.13.1sup_13

20 Sasaki S, Kobayashi M, Tsugane S, JPHC. Validity of a selfadministered food frequency questionnaire used in the 5-year followup survey of the JPHC Study Cohort I: comparison with dietary records for food groups. J Epidemiol 2003;13(Suppl):S57-63. doi:10.2188/ jea.13.1sup_57

21 Ishihara J, Sobue T, Yamamoto S, et al, JPHC. Validity and reproducibility of a self-administered food frequency questionnaire in the JPHC Study Cohort II: study design, participant profile and results in comparison with Cohort I. J Epidemiol 2003;13(Suppl):S134-47. doi:10.2188/jea.13.1sup 134

22 Sasaki S, Ishihara J, Tsugane S, JPHC. Reproducibility of a selfadministered food frequency questionnaire used in the 5-year follow-up survey of the JPHC Study Cohort I to assess food and nutrient intake. J Epidemiol 2003;13(Suppl):S115-24. doi:10.2188/ jea.13.1sup_115
23 Nakaya T, Honjo K, Hanibuchi T, et al, Japan Public Health Center-based Prospective Study Group. Associations of all-cause mortality with census-based neighbourhood deprivation and population density in Japan: a multilevel survival analysis. PLOS One 2014;9:e97802. doi:10.1371/journal.pone.0097802

24 Miki Y, Inoue M, Ikeda A, et al, JPHC Study Group. Neighborhood deprivation and risk of cancer incidence, mortality and survival: results from a population-based cohort study in Japan. PLoS One 2014;9:e106729. doi:10.1371/journal.pone.0106729

25 Berglund P, Heeringa SG. Multiple imputation of missing data using SAS. SAS Institute, 2014. https://support.sas.com/content/dam/ SAS/support/en/books/multiple-imputation-of-missing-data-usingsas/65370 excerpt.pdf.

26 Kato M, Noda M, Inoue M, Kadowaki T, Tsugane S, JPHC Study Group. Psychological factors, coffee and risk of diabetes mellitus among middle-aged Japanese: a population-based prospective study in the JPHC study cohort. Endocr J 2009;56:459-68. doi:10.1507/endocrj. K09E-003

27 Kabeya Y, Kato M, Isogawa A, et al. Descriptive epidemiology of diabetes prevalence and $\mathrm{HbA} 1 \mathrm{c}$ distributions based on a selfreported questionnaire and a health checkup in the JPHC diabetes study. J Epidemiol 2014;24:460-8. doi:10.2188/jea.JE20130196

28 Nanri A, Mizoue T, Shimazu T, et al, Japan Public Health Center-Based Prospective Study Group. Dietary patterns and all-cause, cancer, and cardiovascular disease mortality in Japanese men and women: The Japan public health center-based prospective study. PLoS One 2017;12:e0174848. doi:10.1371/journal.pone.0174848

29 World Health Organization. Prevention of cardiovascular disease: Guidelines for assessment and management of total cardiovascular risk. 2007. https://www.who.int/cardiovascular_diseases/ guidelines/Full\%20text.pdf

30 The Council for Science and Technology; Ministry of Education, Culture, Sports, Science, and Technology; the Government of Japan. Standard tables of food composition in Japan. Fifth Revised and Enlarged Edition, 2005

31 Kokubo Y, Iso H, Ishihara I, Okada K, Inoue M, Tsugane S, JPHC Study Group. Association of dietary intake of soy, beans, and isoflavones with risk of cerebral and myocardial infarctions in Japanese populations: the Japan Public Health Center-based (JPHC) study cohort I. Circulation 2007;116:2553-62. doi:10.1161/ CIRCULATIONAHA.106.683755

32 Zamora-Ros R, Jiménez C, Cleries R, et al. Dietary flavonoid and lignan intake and mortality in a Spanish cohort. Epidemiology 2013;24:726-33. doi:10.1097/ EDE.0b013e31829d5902

33 Talaei M, Koh WP, van Dam RM, Yuan JM, Pan A. Dietary soy intake is not associated with risk of cardiovascular disease mortality in Singapore Chinese adults. J Nutr 2014;144:921-8. doi:10.3945/ jn.114.190454

34 Ministry of Health Law. Japan. National Health and Nutrition Survey, 2015. 2015. https://www.mhlw.go.jp/content/000451759.pdf

35 Shu XO, Zheng Y, Cai H, et al. Soy food intake and breast cancer survival. JAMA 2009:302:2437-43. doi:10.1001/jama.2009.1783

36 Zhang FF, Haslam DE, Terry MB, et al. Dietary isoflavone intake and all-cause mortality in breast cancer survivors: The Breast Cancer Family Registry. Cancer 2017;123:2070-9. doi:10.1002/cncr.30615

37 Kurahashi N, Inoue M, Iwasaki M, Tanaka Y, Mizokami M, Tsugane S, JPHC Study Group. Isoflavone consumption and subsequent risk of hepatocellular carcinoma in a population-based prospective cohort of Japanese men and women. Int / Cancer 2009:124:1644-9. doi:10.1002/ijc.24121

38 Yamamoto S, Sobue T, Kobayashi M, Sasaki S, Tsugane S, lapan Public Health Center-Based Prospective Study on Cancer Cardiovascular Diseases Group. Soy, isoflavones, and breast cancer risk in Japan. J Natl Cancer Inst 2003;95:906-13. doi:10.1093/ jnci/95.12.906

39 Taku K, Lin N, Cai D, et al. Effects of soy isoflavone extract supplements on blood pressure in adult humans: systematic review and meta-analysis of randomized placebo-controlled trials. / Hypertens 2010;28:1971-82. doi:10.1097/ HJH.Ob013e32833c6edb

40 Taku K, Umegaki K, Sato Y, Taki Y, Endoh K, Watanabe S. Soy isoflavones lower serum total and LDL cholesterol in humans: a meta-analysis of 11 randomized controlled trials. Am J Clin Nutr 2007;85:1148-56. doi:10.1093/ajcn/85.4.1148

41 Park Y, Subar AF, Hollenbeck A, Schatzkin A. Dietary fiber intake and mortality in the NIH-AARP diet and health study. Arch Intern Med 2011;171:1061-8. doi:10.1001/archinternmed.2011.18

42 Eisenberg T, Abdellatif M, Schroeder S, et al. Cardioprotection and lifespan extension by the natural polyamine spermidine. Nat Med 2016;22:1428-38. doi:10.1038/nm.4222

Supplementary information: additional tables 1-6 